\title{
Prior experience affects amodal completion in pigeons
}

\author{
Yasuo Nagasaka, Olga F. Lazareva, ANd Edward A. Wasserman \\ University of Iowa, Iowa City, Iowa
}

\begin{abstract}
In a three-alternative forced-choice task, 4 pigeons were trained to discriminate a target stimulus consisting of two colored shapes, one of which partially occluded the other, from two foil stimuli that portrayed either a complete or an incomplete version of the occluded shape. The dependent measure was the percentage of total errors that the birds committed to the complete foil. At the outset of training, the pigeons committed approximately $50 \%$ of total errors to the complete foil, but as training progressed, the percentage of errors to the complete foil rose. When the pigeons were given a second exposure to the initial set of stimuli, they committed $70 \%$ of total errors to the complete foil, suggesting that they now saw the complete foil as more similar to the occluded target than the incomplete foil. These results suggest that experience with 2-D images may facilitate amodal completion in pigeons, perhaps via perceptual learning.
\end{abstract}

"Everything we see hides another thing, we always want to see what is hidden by what we see."

\section{-René Magritte}

Our visual world is filled with objects that are partially obscured and cannot be seen in their entirety. A key task in the daily life of humans and other animals is to identify objects from such incomplete visual information. One of the possible mechanisms for recognizing occluded objects is visual, or amodal, completion: the ability to perceive partially occluded objects as complete, including their shape, texture, and color (Kanizsa, 1979; Palmer, 1999). Most of the published research on amodal completion has used 2-D images; however, several recent reports suggest that many aspects of visual grouping and segmentation involve 3-D information (see Kellman, Garrigan, \& Shipley, 2005 , for an overview). Although the mechanisms of amodal completion may be better understood by using 3-D stimuli, our article focuses on a more basic question: Do pigeons complete a partially occluded shape in 2-D images, just as humans and a number of other species have been shown to do? Therefore, the present study employs 2-D images, which have been used in most previous amodal completion studies.

Several comparative projects have found that not only humans, but also many familiar laboratory animals use amodal completion to identify partially occluded objects shown in 2-D displays (for mice, see Kanizsa, Renzi, Conte, Compostela, \& Guerani, 1993; for Bengalese finches, Okanoya \& Takahashi, 2000; for chickens, Forkman, 1998; Forkman \& Vallortigara, 1999; Lea, Slater, \& Ryan, 1996; Regolin \& Vallortigara, 1995; for squirrel monkeys, Nagasaka \& Osada, 2000; for Japanese macaques, Sugita, 1999; for rhesus macaques, Bakin, Nakayama, \& Gilbert, 2000; Fujita, 2001; Osada \& Schiller, 1994; for baboons, Deruelle, Barbet, Dépy, \& Fagot, 2000; Fagot \& Barbet, 2006; and for chimpanzees, Sato, Kanazawa, \& Fujita, 1997). Research with pigeons, however, has surprisingly yielded no clear evidence of amodal completion (Aust \& Huber, 2006; Cerella, 1980; DiPietro, Wasserman, \& Young, 2002; Fujita, 2001; Fujita \& Ushitani, 2005; Sekuler, Lee, \& Shettleworth, 1996; Shimizu, 1998; Ushitani \& Fujita, 2005; Ushitani, Fujita, \& Yamanaka, 2001; Watanabe \& Furuya, 1997).

It might indeed be the case that, unlike mammals, pigeons and other birds are unable to amodally complete partially occluded stimuli. Yet some avian species (finches and chickens) have exhibited amodal completion in experimental studies, whereas pigeons have not.

Here, it is important to appreciate that many of these comparative studies entailed very different behavioral approaches, making direct comparison difficult, if not impossible. For example, in studies with chickens (Regolin \& Vallortigara, 1995), newly hatched birds were reared in the presence of a salient object (e.g., a red cardboard triangle), producing imprinting, or a strong social attachment, to the object. Later, the chicks were presented with incomplete versions of the imprinted object or with the imprinted object occluded by another surface. Chicks showed a preference for the occluded object, demonstrating that, to them, the occluded object looked more similar to the imprinted object than did the incomplete object.

Although this observation is intriguing and hints at a species difference in amodal completion, caution is warranted. Unlike chickens, pigeons are normally trained in an operant conditioning chamber, with 2-D stimuli pre-

Y. Nagasaka, nyasuo@brain.riken.jp 
sented on a computer monitor (e.g., Sekuler et al., 1996) rather than being presented with real-world 3-D stimuli. We will later review studies that do provide comparable data on amodal completion in different avian species.

Despite these interpretive difficulties, several explanations have been offered for apparent species differences in amodal completion. Fujita and his coauthors (Fujita, 2001; Fujita \& Ushitani, 2005) have proposed differences in foraging behavior to explain the difference between chickens and pigeons in amodal completion: Pigeons, as grain eaters, may have little need to search for food items behind obstacles, whereas chickens, as worm eaters, may have to search for partially visible prey. However, field studies have disclosed that wild rock pigeons (Columba livia) are not exclusively granivorous; during the early spring, earthworms may constitute as much as $60 \%$ of their crop content (Murton \& Westwood, 1966). Nor is the ancestor of domestic chickens, the red jungle fowl (Gallus gallus), an exclusive insectivore. Red jungle fowl are, in fact, omnivorous; they eat grain, shoots of grass, fruits, and berries, as well as earthworms, grasshoppers, and other insects (Ali, Ripley, \& Roberts, 1980).

Another explanation proposed by Fujita and Ushitani (2005) implicates differences in locomotion between pigeons and chickens as the source of disparities in amodal completion. These authors suggested that birds that frequently fly might be placed under greater evolutionary pressure to keep their weight at a minimum than birds that infrequently fly; hence, the pigeon brain may lack certain structures that allow chickens to amodally complete. It should be noted that red jungle fowl are quite capable of flying, as are pheasants and quail. More importantly, it is likely that the ability to quickly and efficiently recognize occluded objects ought to be essential for the survival of a frequently flying bird as well; it is difficult to imagine why individuals lacking this visual ability would have greater reproductive success than individuals possessing it. In any case, this proposal suggests that all birds that frequently fly (in other words, the majority of birds) ought to be unable to amodally complete.

Two studies provide comparable data on amodal completion in pigeons (Shimizu, 1998) and Bengalese finches (Lonchura striata domestica; Okanoya \& Takahashi, 2000). The males of both species were presented with a videotape recording of a female, and the males' courtship displays served as the dependent measure. Both pigeons and finches produced courtship displays in response to the video image of a female. Then, parts of the video image were occluded by a piece of a cardboard. Finches continued to respond to the video image as if it were intact, no matter which areas were occluded. However, pigeons produced courtship displays only if the head was visible and the body was occluded; if the body was visible and the head was occluded, then the frequency of pigeons' courtship displays was greatly diminished. Notably, the ability of Bengalese finches to amodally complete contradicts both hypotheses offered by Fujita and his coauthors (Fujita, 2001; Fujita \& Ushitani, 2005), because finches are mainly (but not exclusively) granivorous and they frequently fly.
Of course, both of these studies used courtship display as the dependent measure. One might argue that courtship is a highly ritualized and stereotyped behavior that may require specific visual cues to be triggered; hence, the difference between finches and pigeons may reflect the disparity in sign stimuli necessary to trigger courtship rather than any difference in these two species' ability to amodally complete (but see Watanabe \& Furuya, 1997).

Finally, Vallortigara and his colleagues (Regolin, Marconato, \& Vallortigara, 2004; Vallortigara, 2004, 2006) have proposed that differences in the neural representation of the frontal binocular field may explain the differences in amodal completion between pigeons and chickens. However, because typical experiments with pigeons and chickens differ methodologically to a great degree, it is unclear whether the behavioral disparities stem from differences in the neurobiology of the visual system or whether they result from the behavioral procedures that may favor amodal completion in chicks and discourage it in pigeons, as Vallortigara (2006) himself has noted.

Regardless of why so many studies have failed to find evidence of amodal completion in pigeons and yet succeeded with other avian species, recent work suggests that, under certain circumstances, pigeons too may be able to amodally complete partially visible stimuli. Nagasaka, Hori, and Osada (2005) used 2-D displays to train pigeons to peck an opaque vertical rectangle located either in front of an opaque horizontal occluder or behind it. Later, the pigeons were presented with stimuli featuring a 2-D transparent occluder. The condition of interest here is the group of birds that was trained to peck the rectangle behind the occluder. If these pigeons were unable to complete the rectangle behind the occluder, then they should have learned to respond to the stimulus as if it were two short rectangles separated by a long, horizontal rectangle; hence, the birds should have been unable to identify the correct stimulus when the occluder was transparent. Instead, Nagasaka et al. (2005) found that these pigeons identified the depth relationship just as well as the pigeons trained to peck the rectangle placed in front of the occluder, thereby suggesting that the pigeons had completed the occluded object during training.

Another recent study suggests that specific training experience may enhance pigeons' recognition of occluded 2-D objects (DiPietro et al., 2002). In this instance, pigeons that were trained to discriminate four basic, shaded shapes evidenced poor, albeit significantly above-chance, recognition of the same shapes when they were partially occluded by a brick wall. Critically, after the birds had later been trained to recognize those same objects placed on top of the brick wall occluder, their recognition of the occluded objects improved dramatically and was comparable to their performance with intact objects. This improvement also transferred to novel objects that had not undergone on-top training. DiPietro et al. reasoned that the pigeons may have had difficulty segregating an object from an occluder, and that on-top training helped them overcome this difficulty, a possibility confirmed by subsequent experimentation (Lazareva, Wasserman, \& Biederman, 2007). These results, together with the data of Na- 
gasaka et al. (2005), suggest that prior failures to obtain amodal completion in pigeons may be related to pigeons' lack of experience with 2-D stimuli and/or the specific learning histories that were provided in those studies.

Of additional relevance is the fact that humans and other organisms may need experience with partially occluded objects in a 2-D environment before they can recognize them. Newborn (Slater et al., 1990) and 2-month-old (Johnson \& Náñez, 1995) infants tested in the habituation/dishabituation paradigm show no signs of recognizing 2-D, partially occluded objects. By 4 months of age, infants can recognize partially occluded objects in a 2-D environment, but only if the occluded object is moved from side to side (Kellman \& Spelke, 1983). Other work evaluating amodal completion in the context of perceptual grouping found evidence of amodal completion in 7-month-old infants, but 5-monthold infants did not amodally complete occluded surfaces (Kavsek, 2004). These and other studies (reviewed by Johnson, 2003) suggest that human infants' ability to amodally complete occluded objects in 2-D displays may develop gradually during the first few months of life. This developmental course may reflect maturational factors, but it may also arise from infants' multiple experiences with occluded 2-D objects.

Most prior studies with pigeons trained the birds to discriminate a complete shape (e.g., a circle) from an incomplete shape (e.g., a Pac-Man face; see Sekuler et al., 1996).
Then, the birds were presented with the training shape occluded by another surface. The specific training experience (notched vs. nonnotched stimuli) may have affected the pigeons' choice behavior in the later test. Our present study addressed this problem by evaluating pigeons' perception of similarity between occluded, complete, and incomplete objects without explicitly teaching a discrimination between complete and incomplete objects.

\section{The Present Study}

In a classic study, Blough (1982) examined pigeons' perception of similarity among letters of the human alphabet. Blough trained pigeons to discriminate one target letter from two foil letters. Blough's analysis of his pigeons' confusion errors to the distractor letters revealed a similarity matrix that was strikingly similar to that obtained with humans (Podgorny \& Garner, 1979). We adapted this experimental technique for our study of amodal completion.

We too gave pigeons a three-alternative forced-choice discrimination task. Each trio of stimuli included an occluded target stimulus, which was reinforced, plus complete and incomplete foils, each of which was nonreinforced (Figure 1). The numbers of errors committed to the complete and incomplete foils therefore indicate whether the pigeons saw the occluded stimulus as being more similar to the complete shape or the incomplete shape.

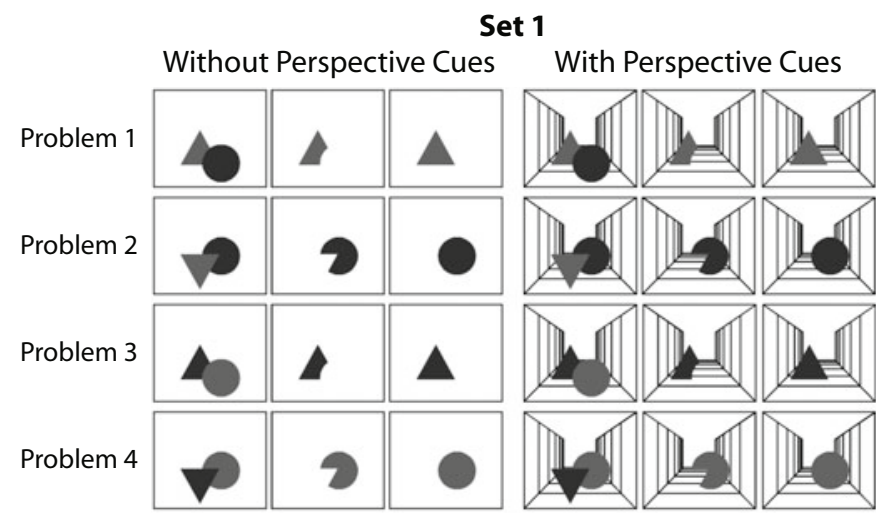

Set 2

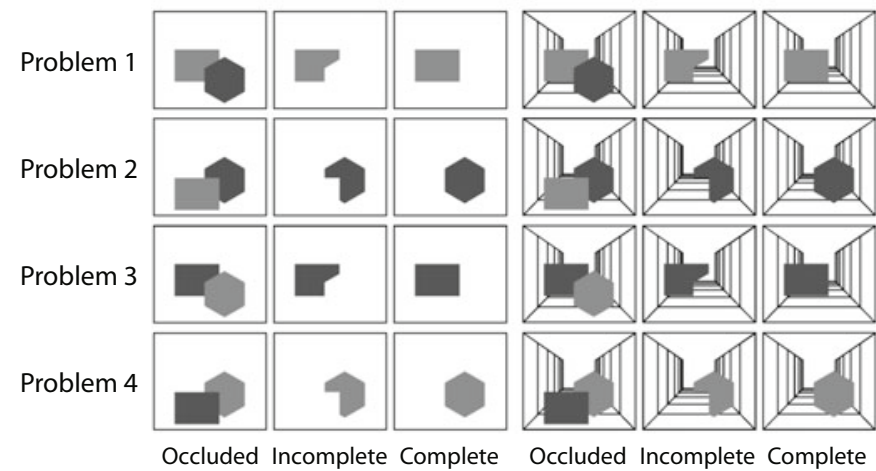

Set 3

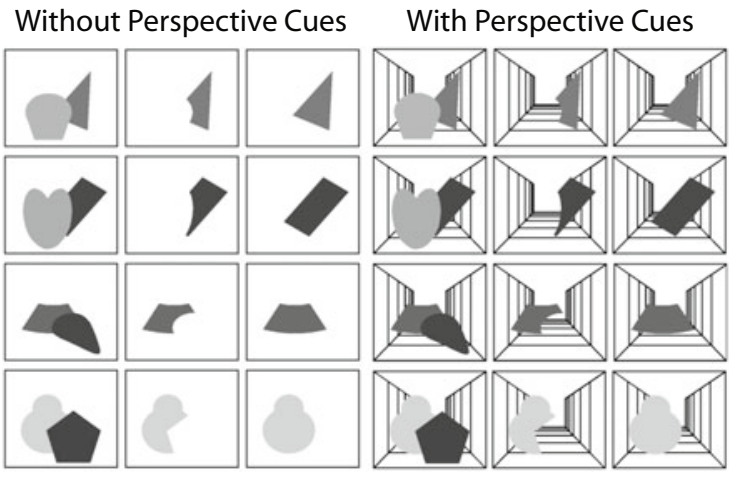

Set 4

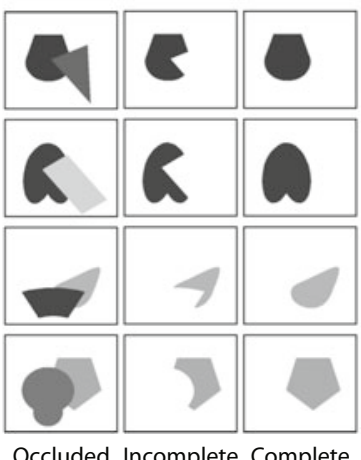

Occluded Incomplete Complete

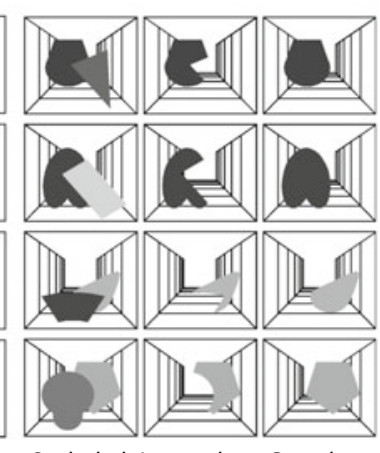

Occluded Incomplete Complete

Figure 1. The four sets of stimuli used in the experiment. Each set consisted of four problems shown either on a white background or with perspective cues. The orientation, location, and depth relation between the objects were counterbalanced in each set. The full set of stimuli in color can be seen at www.psychology.uiowa.edu/Faculty/Wasserman/PigeonAC/Figure1.jpg. 
We also explored the effect of pictorial depth cues on pigeons' performance in this task, because it is known that amodal completion is facilitated by supplementary depth information in adult humans (Nakayama, Shimojo, \& Silverman, 1989) and in nonhuman primates (Sugita, 1999). One of these cues, linear perspective (the perceptual convergence of lines that are parallel in the scene), has been reported to facilitate amodal completion in baboons (Fagot \& Barbet, 2006). Moreover, a recent study found that pigeons can use a number of monocular cues-including occlusion, relative size, and texture gradient - to judge depth in pictorial stimuli (Cavoto \& Cook, 2006). Hence, we tested the effect of pictorial depth cues by creating stimuli that placed complete, incomplete, and occluded objects on a perspective grid (see Figure 1).

\section{METHOD}

\section{Subjects}

The subjects were 4 feral pigeons (Columba livia) kept at $85 \%$ of their free-feeding weights by controlled daily rations. The pigeons had earlier participated in unrelated studies. Bird 17B participated in experiments that used black-and-white Macintosh icons or colored shapes as stimuli; Bird 23Y was exposed to Macintosh icons, photographs of human faces, color clip-art images, and grayscale shaded images; Bird 57B was exposed to colored shapes, grayscale shaded images, and Macintosh icons; and finally, Bird 58W was shown grayscale shaded images, Macintosh icons, clip-art images, and colored shapes. Although the different birds had participated in different experiments before this study, all of them showed the same pattern of responding (see the Results section), suggesting that participating in different prior experiments did not differentially affect their ability to amodally complete

\section{Apparatus}

The pigeons were trained in four operant boxes detailed by B. M. Gibson, Wasserman, Frei, and Miller (2004). The boxes were located in a dark room with continuous white noise. The stimuli were presented on a 15-in. LCD monitor located behind an AccuTouch resistive touch screen (Elo TouchSystems, Menlo Park, CA). A food cup was centered on the rear wall, level with the floor. A food dispenser delivered 45-mg Noyes food pellets through a vinyl tube into the cup. A houselight on the rear wall provided illumination during the session. Each chamber was controlled by an Apple eMac computer. The experimental procedure was programmed in HyperCard (Version 2.4, Apple Computer, Inc., Cupertino, CA).

Three $4.3 \times 3.8 \mathrm{~cm}$ rectangles, or buttons, in the middle of the screen contained the displayed stimuli. The distance between the buttons was $0.8 \mathrm{~cm}$; the distances from the leftmost button to the top of the screen and to the left edge of the screen were $7.7 \mathrm{~cm}$ and $8.3 \mathrm{~cm}$, respectively. The rest of the screen was black.

\section{Stimuli}

Each stimulus consisted of one or two shapes placed on a white background $(4.3 \mathrm{~cm}$ high $\times 3.9 \mathrm{~cm}$ wide). We used a total of four sets of stimuli (shown in Figure 1), each set consisting of four problems. Each problem within a set could be shown on a white background or on a background with perspective cues. The colors of the shapes and the shapes themselves varied from set to set. All of the stimuli that were used in this study can be seen in color at www .psychology.uiowa.edu/Faculty/Wasserman/PigeonAC/Figure1.jpg.

Each problem consisted of three stimuli that were simultaneously presented during training (see below). The occluded stimuli (which ranged from 2,735 to 5,826 pixels) consisted of two colored shapes, one of which occluded the other. Both complete (which ranged from 1,123 to 3,858 pixels) and incomplete (which ranged from 833 to 2,729 pixels) stimuli consisted of a single shape that was partially covered on the corresponding occluded stimulus (e.g., the triangle in the top left row of Figure 1): The complete stimulus showed a full shape, whereas the incomplete stimulus showed the same portion of the shape that was visible on the occluded stimulus. The color, orientation, and depth relation of the shapes were balanced within a set. For example, the triangle in Set 1 could be red or blue, it could be placed in front of the circle or behind the circle, and it could be placed on the left or the right side of the circle.

\section{Procedure}

Pretraining. During pretraining, the birds were taught to peck a square that was placed in the center of the display or at one of four smaller squares that were placed around the central square. The birds then proceeded to training.

Training. Each bird was trained with all four sets of stimuli, both with and without perspective cues, in eight consecutive stages. The order of set presentation was counterbalanced across the birds. Table 1 shows the sequence of set presentation for all 4 birds. Note that 2 of the birds were first exposed to problems with perspective cues, whereas the other 2 birds began the problems without perspective cues. For statistical analyses, each of the two consecutive stages were combined into a single block; thus, each block contained one problem with perspective cues and one problem without perspective cues. Each of the eight consecutive stages consisted of two parts: nondifferential training and differential training, described below.

Nondifferential training. To ensure that the birds were responding similarly to all of the stimuli before differential training, we first conducted nondifferential training. Figure 2 (left panel) shows the sequence of events in the course of a trial. The trial began with the presentation of a black cross centered on a white square $(7 \times 7 \mathrm{~cm})$ in the middle of the display. After a peck at the square, a single

Table 1

Number of Training Sessions to Criterion for Each Bird in Each Stage of Differential Training

\begin{tabular}{|c|c|c|c|c|c|c|c|c|c|}
\hline \multirow[b]{3}{*}{ Block } & \multirow[b]{3}{*}{ Stage } & \multicolumn{8}{|c|}{ Bird } \\
\hline & & \multicolumn{2}{|c|}{ 17B } & \multicolumn{2}{|c|}{$23 \mathrm{Y}$} & \multicolumn{2}{|c|}{$57 \mathrm{~B}$} & \multicolumn{2}{|c|}{$58 \mathrm{~W}$} \\
\hline & & Stimulus & Sessions & Stimulus & Sessions & Stimulus & Sessions & Stimulus & Sessions \\
\hline 1 & 1 & Set $1 \mathrm{P}$ & 3 & Set $1 \mathrm{~N}$ & 3 & Set $2 \mathrm{P}$ & 4 & Set $2 \mathrm{~N}$ & 6 \\
\hline & 2 & Set $2 \mathrm{~N}$ & 7 & Set $2 \mathrm{P}$ & 2 & Set $1 N$ & 2 & Set $1 \mathrm{P}$ & 4 \\
\hline 2 & 3 & Set $1 \mathrm{~N}$ & 4 & Set $1 P$ & 3 & Set $2 N$ & 4 & Set $2 \mathrm{P}$ & 7 \\
\hline & 4 & Set $2 \mathrm{P}$ & 3 & Set $2 \mathrm{~N}$ & 2 & Set $1 P$ & 3 & Set $1 \mathrm{~N}$ & 5 \\
\hline 3 & 5 & Set 3P & 4 & Set $4 \mathrm{P}$ & 3 & Set $3 N$ & 2 & Set $4 \mathrm{~N}$ & 17 \\
\hline & 6 & Set $4 N$ & 4 & Set $3 N$ & 2 & Set $4 \mathrm{P}$ & 5 & Set 3P & 10 \\
\hline 4 & 7 & Set $3 N$ & 6 & Set $4 N$ & 2 & Set 3P & 2 & Set $4 \mathrm{P}$ & 3 \\
\hline & 8 & Set $4 \mathrm{P}$ & 6 & Set 3P & 1 & Set $4 N$ & 3 & Set $3 N$ & 5 \\
\hline Second exposure & & Set $1 \mathrm{P}$ & 2 & Set $1 \mathrm{~N}$ & 2 & Set $2 \mathrm{P}$ & 1 & Set $2 \mathrm{~N}$ & 1 \\
\hline
\end{tabular}

Note-P, problems with perspective cues; N, problems without perspective cues. 


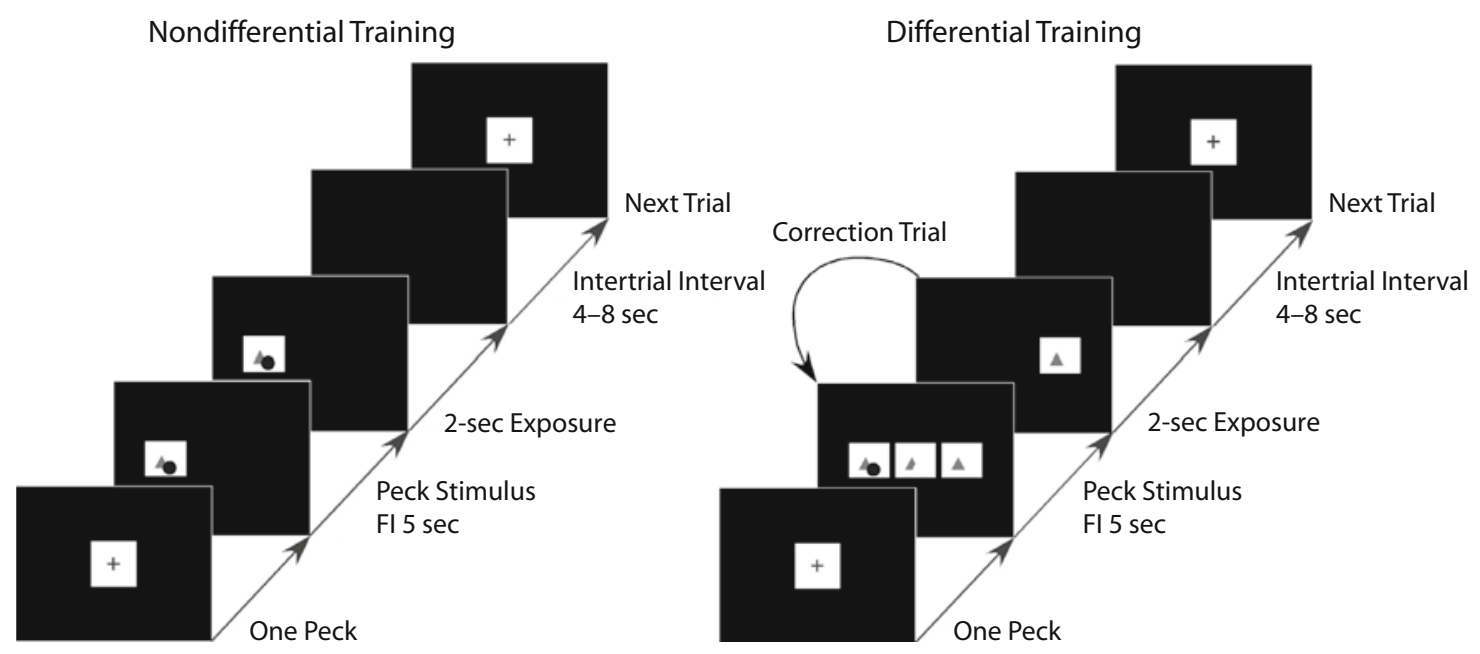

Figure 2. The sequence of events in the course of a trial during nondifferential training (left panel) and differential training (right panel). FI, fixed interval.

stimulus randomly selected from the set later used for differential training appeared for a fixed interval of $5 \mathrm{sec}$. The stimulus could appear in any of the three possible locations (left, middle, or right) on the screen. After the $5 \mathrm{sec}$ had elapsed, the bird had to peck at the stimulus once. After that, the stimulus disappeared, and reinforcement (two food pellets) was delivered with a probability of .5. This probabilistic schedule of reinforcement was introduced during nondifferential training in order to accustom the birds to even lower probabilities of reinforcement during differential training; we wanted to make the task more difficult for the pigeons in order to engender more errors for scoring purposes. The intertrial interval (ITI) lasted from 4 to $8 \mathrm{sec}$; after that, a new trial was given.

Randomizing the location of each stimulus, we obtained a total of 36 unique trials (12 stimuli and three locations). Each session consisted of two blocks of 72 trials (36 reinforced trials and 36 nonreinforced trials), for a total of 144 trials. The birds had to complete two consecutive sessions of nondifferential training before proceeding to differential training.

Differential training. During differential training, all three stimuli that made up the discrimination problem were presented simultaneously, and the bird was required to peck at the occluded stimulus. Figure 2 (right panel) shows the sequence of events in the course of a trial.

The trial began with the presentation of a black cross centered on a white square $(7 \times 7 \mathrm{~cm})$ in the center of the display. After a single peck at the square, three stimuli (occluded, complete, and incomplete) were shown on the screen simultaneously for a fixed interval of $5 \mathrm{sec}$; after that time had elapsed, the pigeons were required to make a single peck at one of the three stimuli. We gave the pigeons $5 \mathrm{sec}$ to view the three-choice stimuli in order to enhance the opportunity for the birds to attend to all of them. The position of each stimulus on the screen (left, middle, right) was randomized within each session. The stimulus to which the bird had responded remained on the screen for $2 \mathrm{sec}$, whereas the other two stimuli were removed; this contingency was arranged to enhance the birds' attending to the stimulus that they had pecked.

If the choice response was correct, then food reinforcement was delivered and the ITI ensued. The probability of food reinforcement after correct trials was set at .33. As mentioned earlier, we used a low probability of reinforcement in order to reduce the pigeons' speed of discrimination learning and hence to increase the number of errors they committed. If the choice response was incorrect, then the screen blackened and a correction trial was given. The ITI randomly ranged from 5 to $13 \mathrm{sec}$ on all trials. Correction trials continued until the correct response was made. Only the first choice response was scored and used in later data analysis, although correction trials were recorded as well. Occasional incomplete sessions were not used in data analysis; such sessions were very rare, and they occurred at the beginning of training.

Randomizing the location of the stimuli within each problem, we obtained 24 unique trials. Thus, the training session consisted of two blocks of 72 trials ( 24 reinforced trials and 48 nonreinforced trials), for a total of 144 trials. Training continued until the birds reached a criterion of $90 \%$ correct within a single session.

Second exposure. After the birds completed the training sequence presented in Table 1, they were exposed to the same set of the stimuli as during Stage 1. Thus, 2 birds were retrained on stimuli with perspective cues and 2 other birds were retrained on stimuli without perspective cues. This retraining was conducted in order to evaluate the effect of the intervening training on the pigeons' distribution of errors. The nondifferential and differential training procedures were the same as described above.

\section{Behavioral Measures}

We used the percentage of errors committed to the complete stimuli (the number of errors to the complete stimuli divided by the total number of errors to both the complete and incomplete stimuli, multiplied by 100) as our dependent measure. For all statistical tests, alpha was set at .05 .

\section{RESULTS}

Table 1 details the number of sessions to criterion for each different training stage for each bird. The discrimination problems proved to be rather easy for the pigeons to master; training to criterion in each stage took a mean of only 4.0 sessions (range: $1-17$ sessions). Also, the speed of acquisition was relatively constant across all stages (with the exception of Bird 58W in Block 3). In other words, the pigeons showed no gradual acquisition of a general rule ("always choose an image consisting of two overlapping objects") from one stimulus set to another. A repeated measures, one-way ANOVA with stage as the independent variable and number of sessions to criterion as the dependent variable supported this observation. The ANOVA found no significant effect of stage $[F(8,24)=$ $\left.1.24, M S_{\mathrm{e}}=6.35, p=.33\right]$, suggesting the absence of a 
general trend across stages. A planned contrast comparing Stage 1 to Stage $8\left[F(1,3)=0.5, M S_{\mathrm{e}}=4.92, p=\right.$ $.836]$ also failed to reach significance, suggesting that there was no measurable increase in the speed of learning from the first problem to the last problem presented during training.

Figure 3 separately depicts the percentage of errors to the complete stimulus over the four training blocks for problems with and without perspective cues. The perspective cues appear to have had no measurable effect on the birds' preference for the complete stimulus (two-way $t$ test, $\left.t_{3}=-0.18, p=.87\right)$. Of special interest was the fact that the percentage of errors to the complete stimulus increased with repeated exposure to the different discrimination problems. This increase is especially intriguing because the birds were always reinforced for selecting the occluded target stimulus; they were never reinforced for selecting either the complete or the incomplete foils. So the increase in the percentage of errors committed to the complete stimulus is unlikely to have been directly produced by the pigeons' reinforcement history.

During the first block, the mean percentage of errors to the complete stimulus $(54.0 \%)$ was quite close to the $50 \%$ level expected by chance; in other words, when the pigeons made errors, they chose the complete stimulus nearly as often as they chose the incomplete stimulus. As training continued, the percentage of errors to the complete stimulus increased until it reached a mean of $64.2 \%$ in the fourth block. Table 2 shows the results of binomial tests for each of the pigeons in each of the four blocks. In the first block, the percentage of errors to the complete stimulus differed significantly from chance for only 1 bird. In the second and third blocks, 2 birds committed

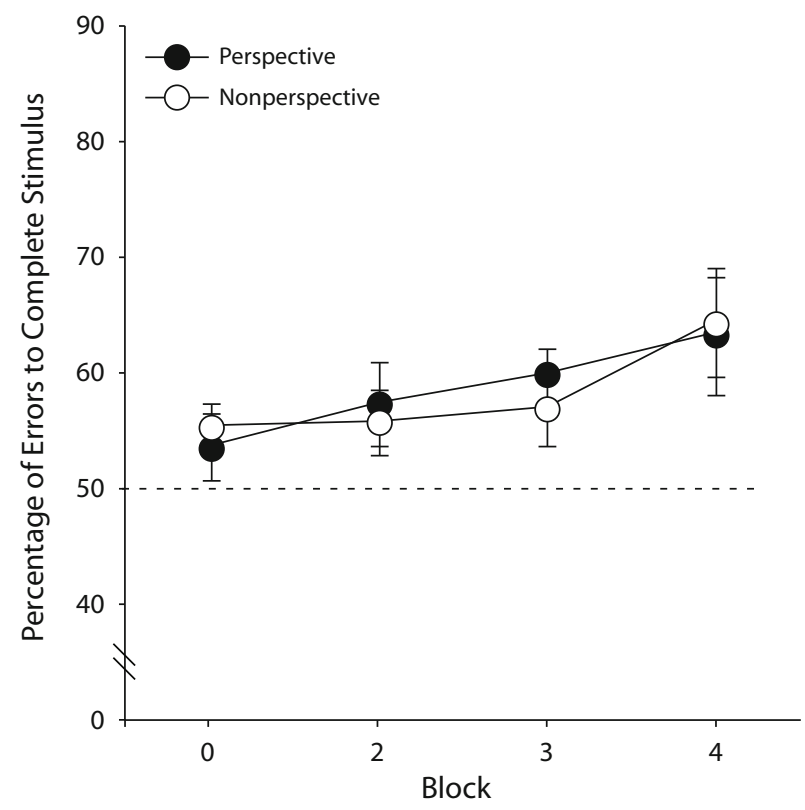

Figure 3. The percentage of total errors committed to the complete stimuli during the four blocks of training. Error bars represent the standard error of the mean (SEM).
Table 2

Number of Errors to Complete and Incomplete Stimuli, Together With Probability of Observing These or More Extreme Values According to Binomial Test

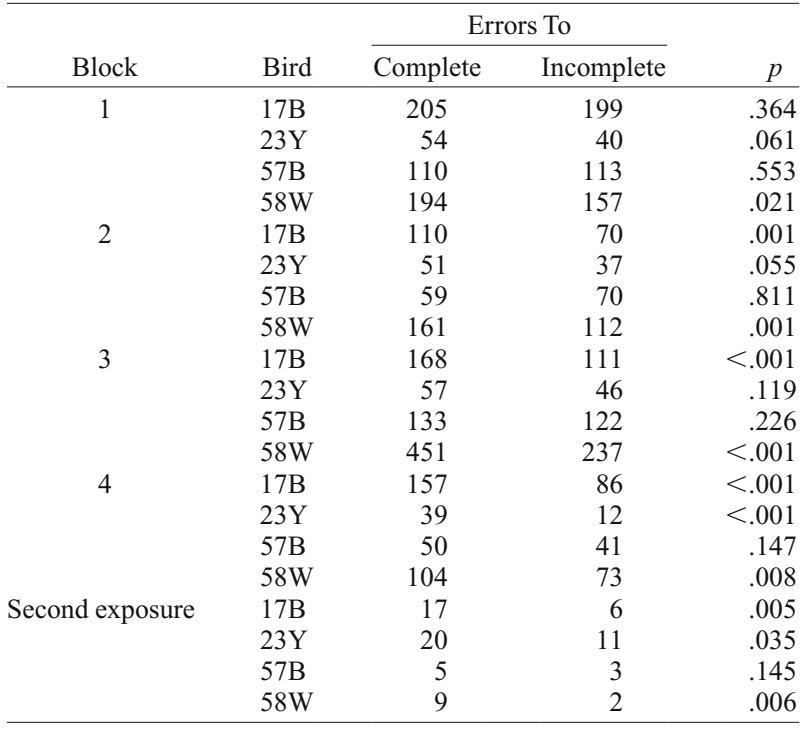

significantly more errors to the complete stimulus than would be expected by chance. Finally, in the fourth block, 3 out of the 4 birds selected the complete stimulus at significantly above-chance levels.

After the birds had completed all four blocks of training, we again presented them with the same problem as they had been given during Stage 1. Table 2 shows the results of the binomial tests for each of the pigeons during this second exposure. In comparison with the first exposure, the mean number of total errors committed during the second exposure was much lower (158.0 and 18.3 errors, respectively). Nevertheless, 3 of the 4 pigeons selected the complete stimulus significantly more often than expected by chance (Table 2 ). The 4 th bird (57B) committed very few errors; although it selected the complete stimulus 5 times out of 8 total errors $(62.5 \%)$, this value was not significantly different from chance.

Figure 4 compares the results of the first exposure and the second exposure. Importantly, the mean percentage of errors committed to the complete stimulus increased from $50.7 \%$ during the first exposure to $70.7 \%$ during the second exposure. A two-tailed paired $t$ test revealed that the percentage of errors to the complete stimulus during the second exposure was significantly higher than during the first exposure $\left(t_{3}=-5.66, p=.01\right)$.

To summarize, we found that, at the beginning of training, the pigeons had no clear preference for either the complete or incomplete foils. With increasing exposure to the different visual discrimination problems, however, the birds began making more errors to the complete than to the incomplete stimulus, suggesting that they now viewed the complete stimulus as more similar to the occluded stimulus than the incomplete stimulus. This finding supports pigeons' amodal completion of 2-D visual stimuli after suitable experience. 


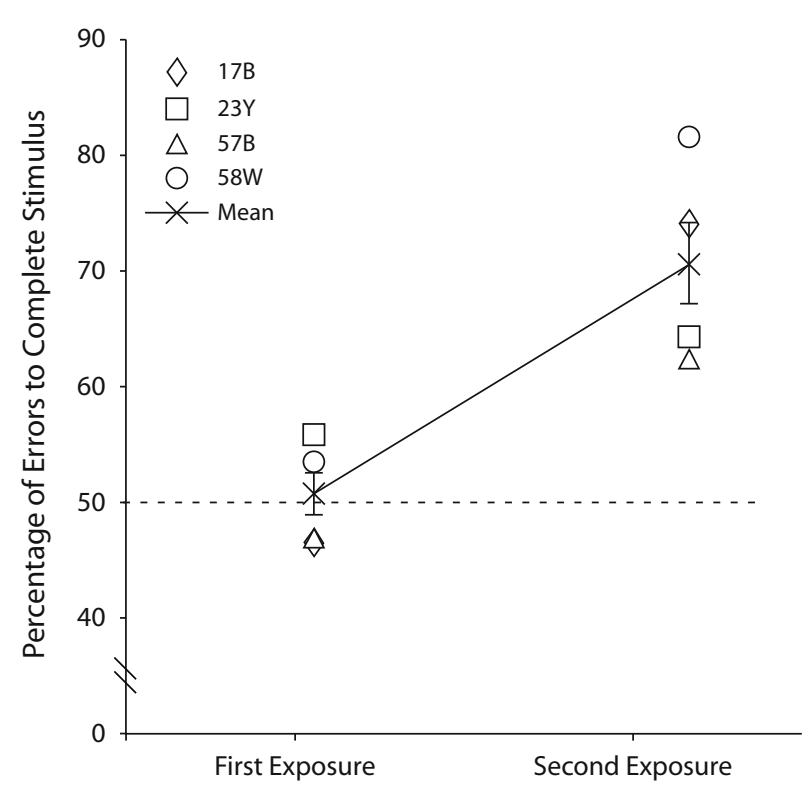

Figure 4. The percentage of total errors committed to the complete stimuli during the first exposure (Stage 1) and the second exposure to the same stimuli. Error bars represent the standard error of the mean $(S E M)$.

\section{DISCUSSION}

In the present experiment, we trained pigeons to perform a simple visual discrimination task: When an occluded stimulus, a complete stimulus, and an incomplete stimulus were presented, the birds were to select the occluded stimulus. This task did not require the birds to segregate the two objects depicted in the occluded stimulus or to complete the occluded object.

At the outset of training, the percentage of total errors committed to the complete stimulus did not differ from chance for 3 out of 4 birds, suggesting that the birds viewed the complete and incomplete stimuli as equally similar to the occluded stimulus. As training proceeded, however, the percentage of total errors to the complete stimulus rose for all of the birds. Because the order in which the different sets of stimuli were presented was counterbalanced across pigeons, it is unlikely that this rise in the percentage of completion errors was due to the particular stimuli themselves.

We further addressed this possibility by giving the birds a second exposure to the same set of stimuli as they had seen in the first stage of training. In the first exposure, completion errors represented only $50.7 \%$ of total errors, whereas in the second exposure, completion errors increased to $70.7 \%$ of total errors. In other words, the birds now viewed the complete stimulus as more similar to the occluded stimulus than the incomplete stimulus.

In our study, perspective cues did not appear to facilitate the pigeons' amodal completion responses. In nonhuman primates (Fagot \& Barbet, 2006), perspective cues have been reported to facilitate amodal completion. One might suggest that pigeons are unable to use perspective cues to extract depth information, but a recent study by
Cavoto and Cook (2006) suggests that pigeons can use a number of pictorial depth cues, including a texture gradient, for discriminating depth relationships among objects. Furthermore, earlier studies reporting amodal completion by chicks (Forkman, 1998; Forkman \& Vallortigara, 1999) presented stimuli with perspective cues; those studies did not present stimuli without perspective cues. Thus, it is unclear whether perspective cues may have facilitated amodal completion by chicks. It is, of course, possible that perspective cues may facilitate amodal completion in mammals, but not in birds; this possible disparity might arise from differences in the anatomical and physiological properties of different animals' respective visual systems. Clearly, further studies evaluating the effects of perspective cues on amodal completion in a wide range of species (see, e.g., Timney \& Keil, 1996) are necessary before any firm conclusions can be drawn.

What might account for the shift in the perceived similarity of occluded, complete, and incomplete stimuli? In each of the problems, the pigeons were always nonreinforced for their choice of either the complete or the incomplete foil stimuli, so the contingencies of reinforcement for responding to these two stimuli cannot be responsible for the increasing percentage of errors to the complete stimulus.

Another potential cue is the area of the stimulus. Because the occluded stimulus consisted of two shapes, its area was always larger than that of the complete or the incomplete stimuli, which each entailed only one shape. If the pigeons were using stimulus size as the main dimension of similarity among the stimuli, then they ought to have committed more errors to the complete stimulus, because its area was larger than that of the incomplete stimulus. Arguing against this account is the fact that the difference in size between the complete and incomplete stimuli was quite small (the average area difference between the complete and incomplete objects was only 620 pixels, or $50.22 \mathrm{~mm}^{2}$ ). Also, the pigeons' choice of the complete stimulus regularly rose during training; simple stimulus generalization according to size ought to have been evident from the inception of training. Still, it is possible that the pigeons were not using size as a dimension of similarity from the outset of the training; as training proceeded, the birds may have learned to rely on the size disparity as an effective discriminative cue, which, in turn, could have affected their perception of similarity among the stimuli. If this were the case, then one might expect to see an increase in the percentage of errors committed to the complete stimulus.

There are two further implications of this account, in terms of stimulus size, that are worthy of consideration. First, if size were an effective cue to which pigeons attend, then we would expect that, as training progressed, the number of sessions to reach criterion ought to have decreased. But we found no effect of stage of training on the speed of acquisition (see the Results section), which calls into question the potential role of size as an effective cue.

Second, if size were an effective cue, then we would expect that disparities in the size of the incomplete stimulus, 
the complete stimulus, and the occluded stimulus ought to have had systematic effects on pigeons' errors to the complete stimulus. We thus calculated the percentage of errors that pigeons committed to the complete stimulus for each problem during Block 3, Block 4, and the second exposure (see Table 1) to the initial problem. During these training blocks, we saw the strongest amodal completion behavior from the pigeons. The percentage of errors to the complete stimulus was then correlated with the ratio of the area of the complete stimulus to the area of the incomplete stimulus and with the ratio of the area of the complete stimulus to the area of the occluded stimulus. The first index $\left(\mathrm{R}_{\mathrm{C} / \mathrm{I}}\right)$ reflects the intuition that, as the disparity in the areas of the complete and incomplete stimuli increases, the number of errors to the complete stimulus should also increase; hence, a positive correlation is expected. The second index $\left(\mathrm{R}_{\mathrm{C} / \mathrm{O}}\right)$ reflects the intuition that the number of errors to the complete stimulus ought to rise as its area approaches the area of the occluded, target stimulus; hence, a negative correlation is expected.

We first analyzed the data in Block 4 alone, because, at this point, the pigeons were most inclined to choose the complete stimulus at reliably above-chance levels (cf. Figure 3); this was termed the one-block correlation. We next computed correlation coefficients for Block 3, Block 4, and the second exposure to the initial problem, combined, in order to increase statistical power; this was termed the multiple-block correlation.

Table 3 presents the Spearman correlation coefficients for both indices for each bird as well as across all 4 birds. As expected, the overall single-block $[r=.19, n=32$, $p=.29]$ and multiple-block $[r=.03, n=80, p=.82]$ correlations for the first index were positive, but the absolute values of both coefficients were extremely small and not statistically significant. The overall single-block $[r=$ $.03, n=32, p=.85]$ and multiple-block $[r=.04, n=80$, $p=.97]$ correlations for the second index were positive, instead of the expected negative scores; they too were not statistically significant.

Scrutiny of the individual pigeons' correlations (see Table 3) also fails to make a strong case for stimulus con- trol by size alone. For example, one would expect that the birds that made more errors to the complete stimulus would also show higher correlations with at least one of our indices, because these birds should be more strongly controlled by the disparity in area (cf. Figure 4). Yet Bird $58 \mathrm{~W}$, which committed the highest percentage of errors to the complete stimulus in the second exposure stage, showed the lowest positive correlation coefficient for the first index. Similarly, Bird 57B, which committed the lowest percentage of errors to the complete stimulus, showed the highest negative correlation coefficient for the second index. These results, together with prior considerations (the small difference between the sizes of the complete and incomplete stimuli and the absence of an effect of training stage on the speed of learning), suggest that the pigeons did not rely on size as the dimension of similarity among the training stimuli.

A final approach to explaining our pigeons' behavior invokes the notion of perceptual learning. Perceptual learning is often defined as the change in an organism's ability to extract previously unused information from the environment (E. J. Gibson, 1969; J. J. Gibson \& E. J. Gibson, 1955; Goldstone, 1998, 2003). One of the mechanisms of perceptual learning works by changing representations of the stimuli, so that they become increasingly differentiated from each other. For example, children may treat the separable dimensions of a single stimulus, such as color and size, as if they were fused together. So children under the age of 5 may categorize objects by their overall similarity, grouping a red ellipse with an orange circle rather than a red ellipse with a blue ellipse (Kemler, 1983; Smith, 1985), whereas school-age children may categorize objects by single dimensions, grouping ellipses with ellipses and circles with circles. It appears that, during development, children may shift from perceiving stimuli globally to analytically decomposing them into separate dimensions (but see Burack, Enns, Iarocci, \& Randolph, 2000; Enns \& Kingstone, 1995; Goldstone, 1998; and Smith, 1989, for further discussion).

In a similar fashion, when pigeons were exposed to the first set of stimuli in Stage 1, they may have perceived the

Table 3

Spearman Correlation Coefficients Between the Percentage of Errors to the Complete Stimulus in Block 4 (One-Block Correlation) and in Blocks 3, 4, and the Second Exposure (Multiple-Block Correlation) to the First Problem With Two Indices: The Ratio of the Area of the Complete Stimulus to the Incomplete Stimulus $\left(R_{C / I}\right)$ and the Ratio of the Area of the Complete Stimulus to the Occluded Stimulus $\left(\mathbf{R}_{\mathrm{C} / \mathrm{O}}\right)$

\begin{tabular}{|c|c|c|c|c|c|c|c|c|}
\hline \multirow[b]{3}{*}{ Bird } & \multicolumn{4}{|c|}{$\mathrm{R}_{\mathrm{C} / \mathrm{I}}$} & \multicolumn{4}{|c|}{$\mathrm{R}_{\mathrm{C} / \mathrm{O}}$} \\
\hline & \multicolumn{2}{|c|}{ One Block } & \multicolumn{2}{|c|}{ Multiple Block } & \multicolumn{2}{|c|}{ One Block } & \multicolumn{2}{|c|}{ Multiple Block } \\
\hline & $r$ & $p$ & $r$ & $p$ & $r$ & $p$ & $r$ & $p$ \\
\hline 17B & .48 & .23 & -.07 & .78 & .43 & .29 & .28 & .23 \\
\hline $23 \mathrm{Y}$ & .19 & .65 & .25 & .29 & -.16 & .71 & -.19 & .42 \\
\hline $57 \mathrm{~B}$ & .21 & .61 & .09 & .70 & -.17 & .69 & -.05 & .83 \\
\hline $58 \mathrm{~W}$ & .17 & .70 & -.11 & .64 & -.19 & .65 & .01 & .97 \\
\hline All birds & .19 & .29 & .03 & .82 & .03 & .85 & .04 & .97 \\
\hline
\end{tabular}

Note-If the birds' performance is controlled by area, then we would expect a positive correlation with $\mathrm{R}_{\mathrm{C} / \mathrm{I}}$ and a negative correlation with $\mathrm{R}_{\mathrm{C} / \mathrm{O}}$. For individual bird correlations, all one-block correlations had a sample size of 8 , and all multiple-block correlations had a sample size of 20 ; for overall correlations, the sample sizes were 32 and 80 , respectively. 
occluded stimulus as a single, fused shape. This proposal is quite plausible, because earlier research has found that pigeons may indeed experience difficulties segregating a pair of 2-D objects when one of them occludes the other (DiPietro et al., 2002; Lazareva et al., 2007). Moreover, edge classification and boundary assignment are required in the early stage of amodal completion (Kellman, Guttman, \& Wickens, 2001; Nakayama et al., 1989); thus, pigeons' inability to segregate the display into two objects would lead to no preference for either the complete or the incomplete shape.

Why would repeated exposure to two similar (but not identical) objects facilitate segregating each from the other? In all of the present problems, our pigeons were required to discriminate an occluded target stimulus (in which one shape partially occluded another) from a complete stimulus and an incomplete stimulus (each of which showed the single shape that had been occluded). This repeated comparison could have been vital to teaching the pigeon to see two separate objects in the occluded stimulus rather than one fused object. Importantly, our procedure ought not to have biased the pigeons toward committing more errors to the complete stimulus than to the incomplete stimulus; it should only have biased the birds toward seeing two objects in the occluded stimulus rather than one. After the birds began segregating the occluded display into two objects - the occluding stimulus and the occluded stimulus - they could have perceived the occluded stimulus as being either more similar to the incomplete stimulus or the complete stimulus, depending on whether their visual system was capable of amodal completion. Our pigeons' results suggest that they came to perceive the occluded stimulus as being more similar to the complete stimulus, suggesting the presence of amodal completion.

These results thus corroborate and extend previous research on amodal completion in pigeons. Although a small number of studies reported that pigeons classify occluded objects as incomplete (Sekuler et al., 1996; Ushitani \& Fujita, 2005, Experiments 1 and 3), most prior studies have found no tendency for pigeons to classify occluded objects as either complete or incomplete (Cerella, 1980; Ushitani \& Fujita, 2005, Experiment 2; Watanabe \& Furuya, 1997). In general, pigeons do not see an occluded stimulus as incomplete (which would constitute strong evidence against amodal completion); instead, they see the occluded stimulus as equally similar to the complete and the incomplete stimuli.

Our pigeons too showed no strong initial preference for choosing the complete or the incomplete stimulus at the beginning of training. However, unlike in previous projects, we continued to expose our pigeons to several different discrimination problems involving stimuli of various shapes and colors; by the end of training, $70.7 \%$ of our birds' errors were committed to the complete stimulus.

At the moment, we do not know whether other kinds of prior experiences (e.g., explicit training to discriminate objects placed in front of the occluder, as in DiPietro et al., 2002) may similarly facilitate amodal completion in pigeons. We also do not know whether all of the pre- vious failures to find amodal completion in pigeons can be explained by appealing to their inability to segregate 2-D objects without prior experience. Finally, we do not know whether pigeons' difficulty with object segregation is due to the lack of prior experience with 2-D images or whether it stems from differences in the anatomical and physiological nature of their visual system (see Regolin, et al., 2004; Vallortigara, 2004, 2006, for a possible neural mechanism underlying the difficulty of finding amodal completion in pigeons). It is clear, however, that repeated exposure to occluded, 2-D stimuli in our study facilitated pigeons' exhibiting amodal completion, presumably by alleviating their problems with object segregation. The dramatic differences in amodal completion by pigeons and mammals (or other birds) may thus be traceable to the types of behavioral tasks or prior experiences the animals have, rather than to their evolutionary origins. Further comparative research is needed to address this intriguing possibility.

\section{AUTHOR NOTE}

This research was supported by National Institute of Mental Health Grant MH47313. We thank Michelle Miner for her assistance in editing this article and in collecting the data. We also thank Leyre Castro and Andrea Frank for their help in collecting the data. Correspondence concerning this article should be addressed to Y. Nagasaka, Laboratory for Symbolic Cognitive Development, RIKEN Brain Science Institute, 2-1 Hirosawa, Wako City, Saitama 351-198, Japan (e-mail: nyasuo@ brain.riken.jp).

\section{REFERENCES}

Ali, S., Ripley, D. S., \& RoberTs, T. J. (1980). Handbook of the birds of India and Pakistan: Together with those of Bangladesh, Nepal, Sikkim, Bhutan and Sri Lanka. Volume 2: Megapodes to crab plover (2nd ed.). Delhi, India: Oxford University Press.

Aust, U., \& Huber, L. (2006). Does the use of natural stimuli facilitate amodal completion in pigeons? Perception, 35, 333-349.

Bakin, J. S., NAKayama, K., \& Gilbert, C. D. (2000). Visual responses in monkey areas V1 and V2 to three-dimensional surface configuration. Journal of Neuroscience, 20, 8188-8198.

BLough, D. S. (1982). Pigeon perception of letters of the alphabet. Science, 218, 397-398.

Burack, J. A., Enns, J. T., Iarocci, G., \& Randolph, B. (2000). Age differences in visual search for compound patterns: Long- versus short-range grouping. Developmental Psychology, 36, 731-740.

Cavoto, B., \& Cоoк, R. G. (2006). The contribution of monocular depth cues to scene perception in pigeons. Psychological Science, 17, 628-634.

Cerella, J. (1980). The pigeon's analysis of pictures. Pattern Recognition, 12, 1-6.

Deruelle, C., Barbet, I., Dépy, D., \& Fagot, J. (2000). Perception of partly occluded figures by baboons (Papio papio). Perception, 29, 1483-1497.

DiPietro, N. T., Wasserman, E. A., \& Young, M. E. (2002). Effects of occlusion on pigeons' visual object recognition. Perception, 31, 1299-1312.

Enns, J. T., \& Kingstone, A. (1995). Access to global and local properties in visual search for compound stimuli. Psychological Science, 6, 283-291.

FAGot, J., \& BARBet, I. (2006). Amodal completion by baboons (Papio papio): Contribution of background depth cues. Primates, 47, $145-150$.

Forkman, B. (1998). Hens use occlusion to judge depth in a twodimensional picture. Perception, 27, 861-867.

Forkman, B., \& Vallortigara, G. (1999). Minimization of modal contours: An essential cross-species strategy in disambiguating relative depth. Animal Cognition, 2, 181-185. 
Fujita, K. (2001). Perceptual completion in rhesus monkeys (Macaca mulatta) and pigeons (Columba livia). Perception \& Psychophysics, 63, 115-125.

Fujita, K., \& Ushitani, T. (2005). Better living by not completing: A wonderful peculiarity of pigeon vision? Behavioural Processes, 69 59-66.

Gibson, B. M., Wasserman, E. A., Frei, L., \& Miller, K. (2004). Recent advances in operant conditioning technology: A versatile and affordable computerized touchscreen system. Behavior Research Methods, Instruments, \& Computers, 36, 355-362.

Gibson, E. J. (1969). Principles of perceptual learning and development. New York: Appleton-Century-Crofts.

Gibson, J. J., \& Gibson, E. J. (1955). Perceptual learning: Differentiation or enrichment? Psychological Review, 62, 32-41.

Goldstone, R. L. (1998). Perceptual learning. Annual Review of Psychology, 49, 585-612.

Goldstone, R. L. (2003). Learning to perceive while perceiving to learn. In R. Kimchi, M. Behrmann, \& C. R. Olson (Eds.), Perceptual organization in vision: Behavioral and neural perspectives (pp. 233280). Mahwah, NJ: Erlbaum.

JoHnson, S. P. (2003). Development of fragmented versus holistic object perception. In G. Schwarzer \& H. Leder (Eds.), The development of face processing (pp. 3-17). Ashland, OH: Hogrefe \& Huber.

Johnson, S. P., \& NÁÑEZ, J. E. (1995). Young infants' perception of object unity in two-dimensional displays. Infant Behavior \& Development, 18, 133-143.

Kanizsa, G. (1979). Organization in vision: Essays on Gestalt perception. New York: Praeger.

Kanizsa, G., Renzi, P., Conte, S., Compostela, C., \& Guerani, L. (1993). Amodal completion in mouse vision. Perception, 22, 713-721.

KAVSEK, M. (2004). The influence of context on amodal completion in 5- and 7-month-old infants. Journal of Cognition \& Development, 5, 159-184.

Kellman, P. J., Garrigan, P., \& Shipley, T. F. (2005). Object interpolation in three dimensions. Psychological Review, 112, 586-609.

Kellman, P. J., Guttman, S. E., \& Wickens, T. D. (2001). Geometric and neural models of object perception. In T. F. Shipley \& P. J. Kellman (Eds.), From fragments to objects: Segmentation and grouping in vision (pp. 183-245). New York: Elsevier.

Kellman, P. J., \& Spelke, E. S. (1983). Perception of partly occluded objects in infancy. Cognitive Psychology, 15, 483-524.

KeMLER, D. G. (1983). Exploring and reexploring issues of integrality, perceptual sensitivity, and dimensional salience. Journal of Experimental Child Psychology, 36, 365-379.

Lazareva, O. F., Wasserman, E. A., \& Biederman, I. (2007). Pigeons' recognition of partially occluded objects depends on specific training experience. Perception, 36, 33-48.

Lea, S. E. G., Slater, A. M., \& Ryan, C. M. E. (1996). Perception of object unity in chicks: A comparison with the human infant. Infant Behavior \& Development, 19, 501-504.

Murton, R., \& Westwood, N. (1966). The foods of the Rock Dove and feral pigeon. Bird Study, 13, 130-146.

NagasaKa, Y., Hori, K., \& Osada, Y. (2005). Perceptual grouping in pigeons. Perception, 34, 625-632.

NAGASAKa, Y., \& Osada, Y. (2000). Subjective contours, amodal completion, and transparency in animals. Japanese Journal of Animal Psychology, 50, 61-73.

Nakayama, K, Shimojo, S., \& Silverman, G. H. (1989). Stereoscopic depth: Its relation to image segmentation, grouping, and the recognition of occluded objects. Perception, 18, 55-68.

Okanoya, K., \& TAKahashi, M. (2000). Shikaku-teki hokan e no seitaigaku-teki apuroochi [Ecological approach to visual completion]. Kokoro no hattatsu: Ninchi-teki seicho no kikoo 1999 [Reports of the Grant-in-aid for Scientific Research for Priority Areas] (pp. 34-41).

Osada, Y., \& Schiller, P. H. (1994). Can monkeys see objects under conditions of transparency and occlusion? Investigative Ophthalmology \& Visual Science, 35, 1664.

Palmer, S. E. (1999). Vision science: Photons to phenomenology. Cambridge, MA: MIT Press.

Podgorny, P., \& GARner, W. R. (1979). Reaction time as a measure of inter- and intraobject visual similarity: Letters of the alphabet. Perception \& Psychophysics, 26, 37-52.

Regolin, L., Marconato, F., \& Vallortigara, G. (2004). Hemispheric differences in the recognition of partly occluded objects by newly hatched domestic chicks (Gallus gallus). Animal Cognition, 7, 162-170.

Regolin, L., \& Vallortigara, G. (1995). Perception of partly occluded objects by young chicks. Perception \& Psychophysics, 57, 971-976.

Sato, A., Kanazawa, S., \& Fujita, K. (1997). Perception of object unity in a chimpanzee (Pan troglodytes). Japanese Psychological Research, 39, 191-199.

Sekuler, A. B., Lee, J. A. J., \& Shettleworth, S. J. (1996). Pigeons do not complete partly occluded figures. Perception, 25, 1109-1120.

Shimizu, T. (1998). Conspecific recognition in pigeons (Columba livia) using dynamic video images. Behaviour, 135, 43-53.

Slater, A., Morison, V., Somers, M., Mattock, A., Brown, E., \& TAYLOR, D. (1990). Newborn and older infants' perception of partly occluded objects. Infant Behavior \& Development, 13, 33-49.

Smith, L. B. (1985). Young children's attention to global magnitude: Evidence from classification tasks. Journal of Experimental Child Psychology, 39, 472-491

Smith, L. B. (1989). A model of perceptual classification in children and adults. Psychological Review, 96, 125-144.

Sugita, Y. (1999). Grouping of image fragments in primary visual cortex. Nature, 401, 269-272.

Timney, B., \& KeIL, K. (1996). Horses are sensitive to pictorial depth cues. Perception, 25, 1121-1128.

Ushitani, T., \& Fujita, K. (2005). Pigeons do not perceptually complete partly occluded photos of food: An ecological approach to the "pigeon problem." Behavioural Processes, 69, 67-78.

Ushitani, T., Fujita, K., \& Yamanaka, R. (2001). Do pigeons (Columba livia) perceive object unity? Animal Cognition, 4, 153-161.

VAllortigara, G. (2004). Visual cognition and representation in birds and primates. In L. J. Rogers \& G. Kaplan (Eds.), Comparative vertebrate cognition: Are primates superior to non-primates? (pp. 57-94). New York: Kluwer/Plenum.

VALlORTIGARA, G. (2006). The cognitive chicken: Visual and spatial cognition in a non-mammalian brain. In E. A. Wasserman \& T. R. Zentall (Eds.), Comparative cognition: Experimental explorations of animal intelligence (pp. 53-70). Oxford: Oxford University Press.

Watanabe, S., \& FurUya, I. (1997). Video display for study of avian visual cognition: From psychophysics to sign language. International Journal of Comparative Psychology, 10, 111-127.

(Manuscript received December 15, 2005; revision accepted for publication September 29, 2006. 